\title{
Substance Use End Date
}

National Cancer Institute

\section{Source}

National Cancer Institute. Substance Use End Date. NCI Thesaurus. Code C83140.

The stop date of the substance usage. 